\title{
GAMIFICAÇÃO E EDUCAÇÃO: CONCEITUAÇÃO, ESTADO DA ARTE E AGENDA DE PESQUISA
}

\section{Gamification and Education: Conceptualization, State of the Art and Research Agenda}

Igor de Albuquerque Cieslak*

Keila Renata Moreira Mourão**

Antônio Jorge Paraense da Paixão ${ }^{* * *}$

\begin{abstract}
Resumo: Os games são uma realidade do mundo atual, fazem parte da vida das pessoas, em especial das crianças e dos jovens. A gamificação enquanto função educativa é um excelente método alternativo para propiciar o ensino-aprendizagem do educando, por ser uma metodologia ativa e motivadora, com resultados positivos em todos os artigos revisados que aplicaram metodologias com gamificação no ambiente escolar. Desta forma, o presente artigo destaca a necessidade de se chegar ao estado da arte da gamificação combinada com a educação. Laboriosamente, o estado da arte foi alcançado com metodologia própria, fundada em três indagações a serem respondidas com auxílio de 22 pesquisas revisadas. As citadas indagações a serem respondidas são: (a) qual a conceituação de gamificação? (b) qual importância de pesquisar a gamificação no contexto educacional? e (c) por que a gamificação deve ser utilizada como recurso didático na educação? O primeiro critério situa o leitor quanto à origem e a conceituação da gamificação. A importância de pesquisar a gamificação indica o que torna o assunto relevante dentro do mundo acadêmico. O último questionamento revela o porquê a gamificação é utilizada como recurso didático na educação. Para atingir o estado da arte na área, foi necessário tirar proveito da seleção de periódicos indexados na forma de artigo da plataforma de diretórios de periódicos CAPES/MEC, acolhendo artigos de qualquer ano e de qualquer idioma. Foi utilizada nas buscas a combinação do termo "gamificação" aos termos "educação" e "ensino", assim como seus derivados (ex.: ensino-aprendizagem e recurso didático).
\end{abstract}

Palavras-chave: Gamificação. Educação. Estado da arte.

\footnotetext{
* Mestre em Desenvolvimento Rural e Gestão de Empreendimentos Agroalimentares do Instituto Federal do Pará (IFPA - Campus Castanhal). Professor EBTT do Instituto Federal do Pará (IFPA - Campus Avançado Vigia). Orcid: https://orcid.org/0000-0002-5883-5110. E-mail: igor.cieslak@ifpa.edu.br.

** Doutora em Ecologia Aquática e Pesca pela Universidade Federal do Pará (UFPA). Professora EBTT do Instituto Federal do Pará (IFPA - Campus Avançado Vigia). Orcid: https://orcid.org/0000-0002-8856-223X. E-mail: kmourao@yahoo.com.br.

*** Doutor em Educação pela Pontifícia Universidade Católica do Rio de Janeiro (PUC-Rio). Professor do Programa de Mestrado em Desenvolvimento Rural e Gestão de Empreendimentos Agroalimentares do Instituto Federal do Pará (IFPA - Campus Castanhal). Orcid: https://orcid.org/0000-0002-5311-041X. E-mail: paraense@outlook.com.
} 


\begin{abstract}
The games are a reality in today's world, they are part of people's lives, especially children and young people. The gamification as an educational function is an excellent alternative method to facilitate the teaching-learning process of the student, for being a motivating and active methodology, with positive results in all the articles reviewed that applied methodologies regarding gamification in the school environment. To do so, this article underlines the need to reach the state of the art of gamification combined with education. Laboriously, the state of the art was reached with its own methodology based on three questions to be answered with the aid of 22 reviewed studies. The questions to be answered were: (a) what is the conceptualization of gamification? (b) How important is it to research gamification in the educational context?, and (c) why should gamification be used as a didactic resource in education? The first criterion places the reader on the origin and conception of gamification. The importance of researching gamification indicates what makes the subject relevant within the academic world. The last question reveals why gamification is used as a didactic resource in education. In order to achieve the state of the art in the area, it was necessary to take advantage of the selection of indexed journals, especially as articles on CAPES/MEC journals directory platform, including articles in any year and any language. It was used for searching the combination of the term "gamification" and the terms "education" and "teaching", as well as their derivatives (e.g. teaching-learning, teaching resource).
\end{abstract}

Keywords: Gamification. Education. State of the art.

\title{
1 Introdução e metodologia
}

Os games fazem parte da vida das pessoas, em especial das crianças e dos jovens (PAULA e VALENTE, 2016; ANDRADE, QUESADA e MARTINS, 2016; MARTINS e GIRAFFA, 2016; MEDEIROS et al., 2016). Com a modernização dos celulares e seu baixo custo, jogar games tornou-se uma experiência amplamente difundida, considerando que boa parte das crianças tem acesso a algum game antes de entrar na pré-escola ou na $1^{\mathrm{a}}$ série do ensino fundamental (PAULA e VALENTE, 2016; ANDRADE, QUESADA e MARTINS, 2016; MARTINS e GIRAFFA, 2016; MEDEIROS et al., 2016)

De acordo com o Comitê Gestor da Internet no Brasil (2013), a pesquisa TIC Kids Online Brasil 2012, entrevistou 1.580 crianças e adolescentes entre 9 e 16 anos, em 111 municípios brasileiros, e obteve os seguintes resultados quando o assunto é a dupla internet/games: (a) a quarta atividade mais realizada na internet por elas é jogar games e/ou jogar games com outras pessoas na internet, representando $54 \%$ dos pesquisados (COMITÊ GESTOR DA INTERNET NO BRASIL, 2013, p. 62); (b) a relação jogar games por faixa etária constatou que 59\% das crianças estão na faixa etária de 9-10 anos, 60\% na faixa etária de 1112 anos, 48\% na faixa etária de 13-14 anos e 51\% na faixa etária de 15-16 anos (COMITÊ GESTOR DA INTERNET NO BRASIL, 2013, p. 62); (c) no que tange à regularidade em jogar: $35 \%$ das crianças jogam todos os dias ou quase todos os dias, $45 \%$ jogam uma ou duas vezes por semana e $19 \%$ jogam uma ou duas vezes por mês (COMITÊ GESTOR DA INTERNET NO BRASIL, 2013, p. 137) e; (d) as crianças que jogam pertencem a todas as classes sociais: $63 \%$ das crianças das classes A e B, $50 \%$ da classe C e $52 \%$ das classes D e E (COMITÊ GESTOR DA INTERNET NO BRASIL, 2013, p. 62).

Nos dias atuais, surgem games que transcendem o entretenimento e abordam temáticas educacionais das mais diversas: medicina, engenharias, administração, matemática, física, biologia, química, história, geografia, topografia, idiomas, entre outras (PAULA e VALENTE, 
2016; ANDRADE, QUESADA e MARTINS, 2016; MEDEIROS et al., 2016; GOMES et al., 2018).

A gamificação se tornou um tema atual e relevante, sendo um recurso didático que auxilia na transposição didática dos conteúdos em sala de aula, coadjuvando no desenvolvimento humano em todas as faixas etárias, sendo, ainda, uma metodologia atraente, chamativa, divertida, cativante e interessante na visão dos discentes (PAULA e VALENTE, 2016; ANDRADE, QUESADA e MARTINS, 2016; MARTINS e GIRAFFA, 2016; MEDEIROS et al., 2016; GOMES et al., 2018).

As gerações Y (nascidas entre 1981-1997), Z (nascidas entre 1998-2009) e $\alpha$ (nascidas após 2010) chegam à escola com a facilidade de aprender acessando sites de busca, desprezando muitas das vezes a aula expositiva de um professor e menosprezando materiais didáticos impressos (ex.: livro, apostila, manuais técnicos, etc.). As citadas gerações são digitais, têm acesso a smartphones, tablets, notebooks e computadores conectados à internet; assim sendo, as meras aulas tradicionais causam a dispersão da maior parte dos alunos.

Os discentes estão munidos de informação em sites de busca, como Google, Yahoo, Wikipédia e UOL, e acabam deixando a figura do docente de lado no processo de ensinoaprendizagem se este trouxer as "velhas" metodologias tradicionais para sala de aula.

O lúdico é uma excelente forma de transmitir conhecimentos, habilidades e atitudes de forma descontraída (ALMEIDA, PROCHNOW \& LOPES, 2016; MENDONÇA, 2010; CARVALHO, WAGNER \& QUITETE, 2013; SOARES et al., 2017; GROSSI, 2017; FURLAN et al., 2015; BERTON, USTÁRROZ \& COSTA, 2016; ALVES \& SILVA, 2014; VIEIRA, 2012; HANSTED \& GOHN, 2013). Dentre as suas categorizações, a gamificação é uma categoria recomendada para as gerações $\mathrm{Y}, \mathrm{Z}$ e $\alpha$, considerando serem altamente tecnológicas e passarem boa parte da vida em contato com games (DETERDING et al., 2011; DOMINGUEZ et al., 2013; LANDERS, 2014; RICHTER et al., 2015; CIESLAK, 2018).

Diante do exposto, é possível inferir que a educação é indissociável da gamificação, sendo fundamental discutir a conceituação de gamificação, a sua importância no contexto educacional e o porquê deve ser utilizada na educação.

Desta forma, o objetivo geral desta revisão de literatura foi identificar a conceituação de gamificação e a sua importância no contexto educacional.

Foram desenvolvidos os seguintes objetivos específicos: a) efetuar revisão bibliográfica sobre a conceituação de gamificação; b) verificar a importância de pesquisar a gamificação no contexto educacional; c) identificar o porquê a gamificação deve ser utilizada como recurso didático na educação.

No que tange à metodologia da pesquisa, o presente trabalho pode ser classificado como uma pesquisa teórica e exploratória, com uso de abordagem qualitativa por meio da revisão bibliográfica, a partir de artigos publicados na área da gamificação e educação publicados em qualquer ano e em qualquer idioma.

A seleção dos artigos na temática do presente trabalho guiou-se pelos seguintes passos:

a) Seleção de periódicos indexados na plataforma de diretórios de periódicos CAPES/MEC. Foi utilizado o termo "gamificação" associado aos termos "educação" e "ensino", assim como seus derivados (ex.: ensino-aprendizagem e recurso didático).

b) As buscas ocorreram em 23/04/2018 e foram encontrados 35 registros após a exclusão de artigos duplicados. 
c) A partir da seleção dos trabalhos (Figura 1) que se referem efetivamente ao tema pesquisado a partir da leitura do título, das palavras-chave, do resumo e, em alguns casos, do trabalho na íntegra, buscou-se identificar os artigos que se aproximariam mais do objetivo da pesquisa. Para facilitar esse árduo trabalho, foi construído um roteiro de fichamento com 03 (três) perguntas (conforme Tabela 1, apresentada posteriormente - Indagações no roteiro de fichamento), sendo excluídos os artigos que não respondiam à totalidade das perguntas. Assim, dos 35 registros encontrados no segundo passo, 22 conversavam com a temática desta pesquisa.

Figura 1 - Fluxograma do processo de seleção dos artigos pesquisados. O número de artigos em cada etapa está indicado entre parênteses.

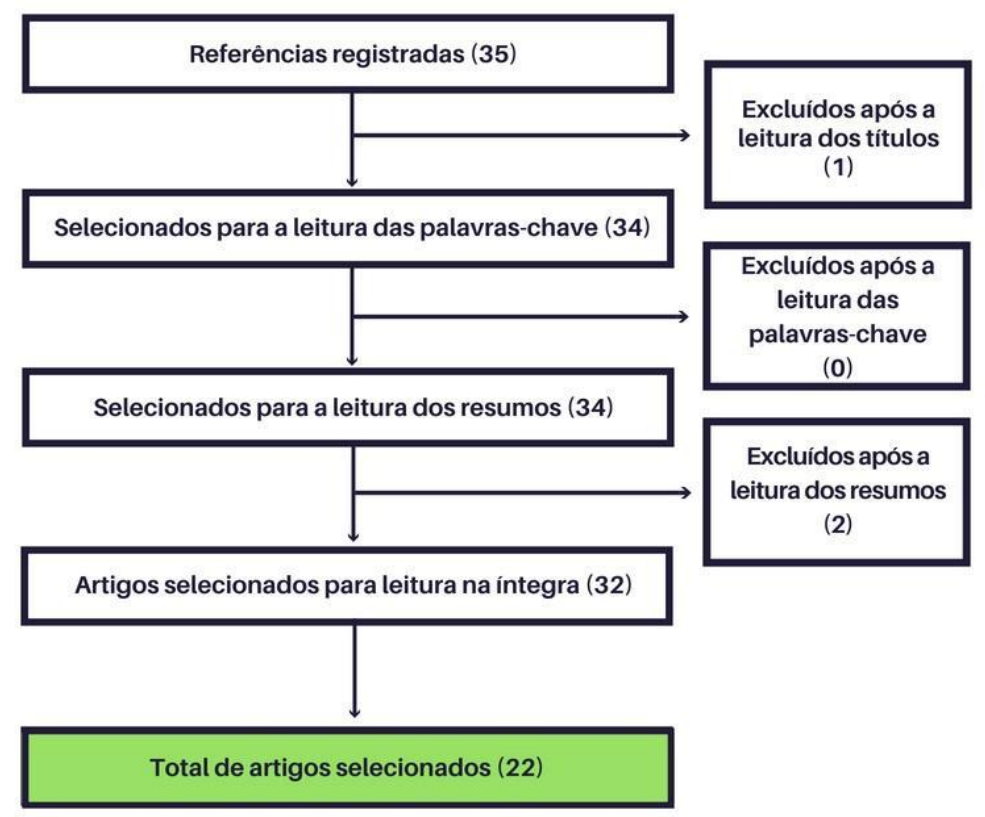

Fonte: Elaborado pelos autores.

Percebeu-se, a partir deste levantamento, que nos últimos anos a produção científica no Brasil tem se avolumado na temática "gamificação e educação", sugerindo que o campo de estudo está em processo de consolidação e se faz relevante do ponto de vista acadêmico e científico.

O trabalho foi dividido em 03 (três) partes: introdução e metodologia, o estado da arte da gamificação AND educação, e conclusão e proposta de agenda de pesquisa. Na primeira parte, realizou-se a justificativa da revisão de literatura, a definição dos objetivos da pesquisa e apresentaram-se os aspectos metodológicos. Na segunda parte, apresentou-se o contexto das publicações, as características metodológicas e os principais resultados encontrados nas pesquisas revisadas. Na terceira e última parte, expôs uma síntese sobre o que se sabe acerca do tema até o momento e quais aspectos não foram estudados para trabalhos futuros, os indícios obtidos, questões em aberto, perspectivas futuras e repassado os objetivos específicos.

\section{0 estado da arte da gamificação AND educação}

Conforme já debruçado na introdução, esta parte apresentou o contexto das publicações, as características metodológicas e os principais resultados encontrados nas pesquisas revisadas. 
Vale reiterar que a metodologia para chegar ao estado da arte adotou três indagações a serem analisadas: (a) qual a conceituação de gamificação? (b) qual importância de pesquisar a gamificação no contexto educacional? e (c) por que a gamificação deve ser utilizada como recurso didático na educação? O primeiro critério situa o leitor quanto à origem da gamificação. A importância de pesquisar a gamificação indica o que torna o assunto relevante dentro do mundo acadêmico. O último questionamento revela por que a gamificação é utilizada como recurso didático na educação.

Feitas as considerações de estilo sobre o presente tópico, vale apresentar os resultados apurados nos 22 artigos selecionados nesta pesquisa.

\subsection{Contexto das publicações}

Percebe-se um aumento quase geométrico no número de publicações sobre gamificação AND educação no período de 2011 a 2018. Nos primeiros dois anos, 2011 a 2012, apenas dois (9\%) estudos foram publicados; de 2013 a 2014, quatro (18\%); de 2015 a 2016, sete (32\%); e no período final, de 2017 a 2018, nove (41\%) estudos foram publicados. Destaca-se o ano de 2017, com 7 publicações. O aumento na quantidade de publicações a respeito do tema pode ser explicado, em parte, pela notoriedade que a nomenclatura "gamification" adquiriu, em 2011, após o lançamento do livro de McGonigal intitulado de "Reality is broken: why games make us better and how they can change the world", somada à percepção dos docentes de que a gamificação aplicada à educação traz benefícios como: motivação, prazer, engajamento, divertimento, facilita o ensino-aprendizagem, além de que os alunos preferem as aulas gamificadas às tradicionais.

Das 22 publicações revisadas, dezenove (86\%) referem-se às temáticas gamificação combinado com educação, três (13\%) referem-se exclusivamente à gamificação e nenhuma $(0 \%)$ refere-se exclusivamente à educação.

Periódicos da área de educação foram os que mais publicaram (77\%) a respeito do tema na presente revisão. Infelizmente, a "ciência da gamificação" é tratada pela Plataforma Sucupira (Capes) como uma subárea dos periódicos das áreas de: educação, ensino, comunicação e informação, ciência da computação, interdisciplinar, linguística e literatura e medicina.

Desta forma, existem apenas 9 periódicos específicos na "ciência da gamificação" na Plataforma Sucupira (Capes): (1) International journal of game-based learning (área: educação - Qualis A2 - ISSN 2155-6849); (2) International journal of computer games technology (área: ciência da computação - Qualis B1 - ISSN 1687-7047); (3) Games for health journal (área: medicina II - Qualis B2 - ISSN 2161-783X); (4) The computer games journal (área: interdisciplinar - Qualis B3 - ISSN 2052-773X); (5) G|A|M|E ? Games as arts, media, entertainment (área: comunicação e informação - Qualis B4 - ISSN 2280-7705); (6) Games for health: research, development, and clinical applications (área: ciência da computação Qualis B5 - ISSN 2161-7856); (7) JMIR serious games (área: ciência da computação - Qualis B5 - ISSN 2291-9279); (8) Anais do simpósio brasileiro de jogos e entretenimento digital (área: interdisciplinar - Qualis: C - ISSN 2179-2259) e; (9) Gamepad (área: interdisciplinar - Qualis C - ISSN 2236-7497). Umas das consequências disso, para a área, é a perda de espaço por omissão, ou seja, um tema que deveria ser desenvolvido essencialmente no âmbito da ciência da gamificação acaba se desenvolvendo em outras áreas. Esta situação tende a ser agravada nos próximos anos, por exemplo, com o interesse cada vez maior das licenciaturas tratarem a temática em ensino-aprendizagem e da medicina encontrar soluções mais econômicas para treinamento de pessoas e cirurgias menos invasivas com uso da gamificação. 


\subsection{Características metodológicas}

Das 22 pesquisas revisadas, quinze (68\%) são qualitativas, zero (0\%) quantitativa, e sete podem ser caracterizadas como mistas, com a utilização de procedimentos quantitativos e qualitativos. Em relação ao tipo de estudo, nove $(41 \%)$ pesquisas foram consideradas descritivas, e uma (5\%) explicativa. Pesquisas exploratórias, que procuram explorar a gamificação na educação para fornecer informações para uma investigação mais precisa, representam mais da metade da amostra revisada, ou seja, doze (54\%).

Diferentes formas de coleta de dados e informações foram exploradas nos estudos revisados. Em $11(50 \%)$ pesquisas, predominou a coleta bibliográfica, 4 (18\%) utilizaram questionários, 3 (14\%) fizeram observações e 4 (18\%) apresentaram relatos.

Quase a totalidade dos artigos selecionados propaga a gamificação como um potencial de ensino, seja por causa do aspecto lúdico, por gerar motivação ou prazer ao estudante, ou por auxiliar na resolução de problemas e no processo de aprendizagem. Todavia, pouco vem sendo abordado sobre a relação da gamificação como ferramenta de avaliação de aprendizagem. $\mathrm{Na}$ presente revisão da literatura, apenas uma pesquisa relacionou a gamificação a um instrumento de avaliação de aprendizagem.

Interessante apontar que das 22 publicações nos últimos oito anos cobertos pela revisão, de 2011 a 2018, em 8 (36\%) pesquisas buscou-se desenvolver games, 3 (14\%) utilizou games já existentes em sala de aula e $11(50 \%)$ tratou o tema exclusivamente sob o ponto de vista bibliográfico. Isso demonstra que mais da metade dos autores que abordam gamificação AND educação não desenvolveram novos games, o que deixa a temática muito superficial, com pouca aplicabilidade e inovação acadêmica.

\subsection{Principais resultados encontrados nas pesquisas revisadas}

Conforme os critérios estipulados na metodologia para seleção de artigos foram redigidas 03 (três) indagações no roteiro de fichamento, de acordo com os termos do Quadro 1.

Quadro 1 - Indagações no roteiro de fichamento

\begin{tabular}{|c|l|}
\hline $\mathbf{N}^{\mathbf{0}}$ & \multicolumn{1}{c|}{ INDAGAÇÕES } \\
\hline 01 & O artigo possui algum trecho que trata sobre: “Conceituação de gamificação”. \\
\hline 02 & $\begin{array}{l}\text { O artigo possui algum trecho que trata sobre: “Qual importância de pesquisar a gamificação no contexto } \\
\text { educacional?”. }\end{array}$ \\
\hline 03 & $\begin{array}{l}\text { O artigo possui algum trecho que trata sobre: "Por que a gamificação deve ser utilizada como recurso } \\
\text { didático na educação?". }\end{array}$ \\
\hline
\end{tabular}

Fonte: Elaborado pelos autores. 
Revista de Educação, Ciência e Tecnologia

O Quadro 2 apresenta os 22 (vinte e dois) artigos selecionados por autor/ano/título.

Quadro 2 - Artigos selecionados por autor/ano e título

\begin{tabular}{|c|c|c|}
\hline $\mathbf{N}^{\mathbf{0}}$ & AUTOR/ANO & TíTULO \\
\hline 01 & COELHO (2011) & Um mapeamento do conceito de jogo \\
\hline 02 & ORLANDI et al. (2018) & Gamificação: uma nova abordagem multimodal para a educação \\
\hline 03 & BUENO \& BIZELLI (2014) & A gamificação do processo educativo \\
\hline 04 & CRUZ JUNIOR (2014) & Burlando o círculo mágico: o esporte no bojo da gamificação \\
\hline 05 & SCHLEMMER (2016) & Games e gamificação: uma alternativa aos modelos de $\mathrm{EaD}$ \\
\hline 06 & $\begin{array}{l}\text { RIBEIRO \& MONTANARO } \\
(2017)\end{array}$ & $\begin{array}{l}\text { A gamificação gamificada: desenvolvimento de um curso para } \\
\text { capacitação de docentes }\end{array}$ \\
\hline 07 & $\begin{array}{l}\text { MATTAR \& NESTERIUK } \\
(2016)\end{array}$ & $\begin{array}{l}\text { Estratégias do design de games que podem ser incorporadas à } \\
\text { educação a distância }\end{array}$ \\
\hline 08 & $\begin{array}{l}\text { SAMPAIO \& BERNARDINO } \\
\text { (2017) }\end{array}$ & $\begin{array}{l}\text { O uso de metodologias alternativas no ensino de biblioteconomia: } \\
\text { gamificação como estratégia pedagógica }\end{array}$ \\
\hline 09 & FRAGELLI (2017) & $\begin{array}{l}\text { Gamificação como um processo de mudança no estilo de ensino } \\
\text { aprendizagem no ensino superior: um relato de experiência }\end{array}$ \\
\hline 10 & $\begin{array}{l}\text { AMÉRICO, NAVARI \& } \\
\text { BELDA (2014) }\end{array}$ & $\begin{array}{l}\text { Gamificação: modelagem de conteúdos para programas e aplicativos } \\
\text { educativos no ambiente da TV digital interativa }\end{array}$ \\
\hline 11 & MARTINS et al. (2016) & $\begin{array}{l}\text { A gamificação no ensino de história: o jogo "Legend of zelda" na } \\
\text { abordagem sobre medievalismo }\end{array}$ \\
\hline 12 & $\begin{array}{l}\text { MONTE, BARRETO \& } \\
\text { ROCHA (2017) }\end{array}$ & Gamification e a web 2.0: planejando processo ensino-aprendizagem \\
\hline 13 & CRUZ JUNIOR (2017) & $\begin{array}{l}\text { Vivendo o jogo ou jogando a vida? Notas sobre jogos (digitais) e } \\
\text { educação em meio à cultura ludificada }\end{array}$ \\
\hline 14 & $\begin{array}{l}\text { CHRISTIANINI, } \\
\text { GRANDE \& AMÉRICO } \\
(2016)\end{array}$ & $\begin{array}{l}\text { Desenvolvimento de sistemas gamificados com foco no } \\
\text { edutretenimento e no jogador: uma análise dos arquétipos de Bartle e } \\
\text { Marczewski }\end{array}$ \\
\hline 15 & MASSI (2017) & $\begin{array}{l}\text { Criação de objetos de aprendizagem gamificados para uso em sala de } \\
\text { treinamento }\end{array}$ \\
\hline 16 & SANDE \& SANDE (2018) & $\begin{array}{l}\text { Uso do Kahoot como ferramenta de avaliação e ensino-aprendizagem } \\
\text { no ensino de microbiologia industrial }\end{array}$ \\
\hline 17 & $\begin{array}{l}\text { ALTHAUS, DULLIUS \& } \\
\text { AMADO (2016) }\end{array}$ & Jogo computacional e resolução de problemas: três estudos de casos \\
\hline 18 & SILVA (2015) & Uso de gamificação como instrumento de avaliação da aprendizagem \\
\hline 19 & CANI et al. (2017) & $\begin{array}{l}\text { Análise de jogos digitais em dispositivos móveis para aprendizagem } \\
\text { de línguas estrangeiras }\end{array}$ \\
\hline 20 & $\begin{array}{l}\text { PAGANINI \& BOLZAN } \\
\text { (2016) }\end{array}$ & Ensinando física através da gamificação \\
\hline 21 & LOPES \& OLIVEIRA (2013) & $\begin{array}{l}\text { Videojogos, serious games e simuladores na educação; usar, criar e } \\
\text { modificar }\end{array}$ \\
\hline 22 & $\begin{array}{l}\text { MARQUES, } \quad \text { SILVA } \\
\text { MARQUES (2011) }\end{array}$ & $\begin{array}{l}\text { A influência dos videojogos no rendimento escolar dos alunos: uma } \\
\text { experiência no } 2^{\circ} \text { e } 3^{\circ} \text { Ciclo do ensino básico }\end{array}$ \\
\hline
\end{tabular}

Fonte: Elaborado pelos autores. 
A seguir, analisam-se os achados destes artigos, considerando-se cada uma das indagações de análise utilizadas no presente estudo.

A Tabela 1 apresenta as principais respostas identificadas para a primeira indagação (o artigo possui algum trecho que trata sobre: "Conceituação de gamificação"):

Tabela 1 - Respostas da primeira indagação

\begin{tabular}{|c|c|c|}
\hline Paráfrase das principais respostas & $\%$ & Autor/Ano \\
\hline $\begin{array}{l}\text { Atividade com regras e normas, que cria uma } \\
\text { realidade estruturada. }\end{array}$ & $27 \%$ & $\begin{array}{l}\text { COELHO (2011); BUENO \& BIZELLI (2014); } \\
\text { SCHLEMMER (2016); MATTAR \& NESTERIUK } \\
\text { (2016); MONTE, BARRETO \& ROCHA (2017); } \\
\text { ALTHAUS, DULLIUS \& AMADO (2016) }\end{array}$ \\
\hline $\begin{array}{l}\text { Utiliza elementos dos games (mecânicas, } \\
\text { estratégias e pensamentos) ou design de } \\
\text { games fora do seu contexto (em situações de } \\
\text { não-game), como na educação, por exemplo. } \\
\text { (Definição de Kapp, 2012). }\end{array}$ & $64 \%$ & $\begin{array}{l}\text { ORLANDI et al. (2018); BUENO \& BIZELLI (2014); } \\
\text { CRUZ JUNIOR (2014); MATTAR \& NESTERIUK } \\
\text { (2016); SAMPAIO \& BERNARDINO (2017); } \\
\text { FRAGELLI (2017); AMÉRICO, NAVARI \& BELDA } \\
\text { (2014); MARTINS et al. (2016); CHRISTIANINI, DE } \\
\text { GRANDE \& AMÉRICO (2016); MASSI (2017); } \\
\text { SANDE \& SANDE (2018); SANDE \& SANDE } \\
\text { (2018); SILVA (2015); CANI et al. (2017) }\end{array}$ \\
\hline $\begin{array}{l}\text { Envolve mecânica própria de pontuação, } \\
\text { ranking ou placar, recompensa, premiação, } \\
\text { medalhas, enigmas, abertura de novos } \\
\text { mapas, missões desafiadoras, evolução do } \\
\text { personagem, conquista de novos poderes ou } \\
\text { skills, e etc. }\end{array}$ & $36 \%$ & $\begin{array}{l}\text { SCHLEMMER (2016); MATTAR \& NESTERIUK } \\
\text { (2016); FRAGELLI (2017); AMÉRICO, NAVARI \& } \\
\text { BELDA (2014); MARTINS et al. (2016); MONTE, } \\
\text { BARRETO \& ROCHA (2017); CHRISTIANINI, DE } \\
\text { GRANDE \& AMÉRICO (2016); SILVA (2015) }\end{array}$ \\
\hline $\begin{array}{l}\text { Conjunto de estratégias num universo } \\
\text { propriamente digital, por meio de mecânicas } \\
\text { de jogos para a resolução de problemas e/ou } \\
\text { desenvolvimento de certos conteúdos, } \\
\text { inclusive educacionais. }\end{array}$ & $9 \%$ & $\begin{array}{l}\text { RIBEIRO \& MONTANARO (2017); LOPES \& } \\
\text { OLIVEIRA (2013) }\end{array}$ \\
\hline $\begin{array}{l}\text { É o uso de games com propostas de } \\
\text { engajamento, jogabilidade, sistema de } \\
\text { recompensas e ensinos colaborativos. } \\
\text { (Definição de McGonigal, 2002). }\end{array}$ & $4 \%$ & MONTE, BARRETO \& ROCHA (2017) \\
\hline $\begin{array}{l}\text { Consiste na escolha e aplicação seletiva de } \\
\text { princípios lúdicos em circunstâncias não } \\
\text { lúdicas. }\end{array}$ & $4^{c}$ & CRUZ JUNIOR (2017) \\
\hline
\end{tabular}

Ferramenta ou tecnologia para o desenvolvimento de soluções educacionais no contexto do edutretenimento.

CHRISTIANINI, DE GRANDE \& AMÉRICO (2016)

$4 \%$

Uma técnica ou processo de pensar como num jogo e as mecânicas de um game para motivar pessoas e resolver problemas. (Definição de Nick Pelling, 2002).

PAGANINI \& BOLZAN (2016)

Combinação de digital, mais lúdico, e em alguns casos, o educativo. 
Na maioria dos estudos revisados (64\%), a conceituação de gamificação utilizou a definição do autor Kapp, que definiu a gamificação como a utilização de elementos dos games (mecânicas, estratégias e pensamentos) ou design de games fora do seu contexto (em situações de não-game), como na educação, por exemplo. Por outro lado, $36 \%$ das pesquisas definiu que gamificação compreende uma mecânica própria de pontuação, ranking ou placar, recompensa, premiação, medalhas, enigmas, abertura de novos mapas, missões desafiadoras, evolução do personagem, conquista de novos poderes ou skills, etc.

As principais respostas identificadas para a segunda indagação (o artigo possui algum trecho que trata sobre: "Qual importância de pesquisar a gamificação no contexto educacional?”) estão na Tabela 2:

Tabela 2 - Respostas da segunda indagação

\begin{tabular}{|c|c|c|}
\hline Paráfrase das principais respostas & $\%$ & Autor/Ano \\
\hline Chamamento e aderência cultural e histórica & $23 \%$ & $\begin{array}{l}\text { COELHO (2011); ORLANDI et al. (2018); BUENO } \\
\text { \& BIZELLI (2014); MARTINS et al. (2016); CRUZ } \\
\text { JUNIOR (2017) }\end{array}$ \\
\hline É uma realidade do mundo atual & $54 \%$ & $\begin{array}{l}\text { ORLANDI et al. (2018); CRUZ JUNIOR (2014); } \\
\text { SAMPAIO \& BERNARDINO (2017); AMÉRICO, } \\
\text { NAVARI \& BELDA (2014); MARTINS et al. (2016); } \\
\text { MONTE, BARRETO \& ROCHA (2017); CRUZ } \\
\text { JUNIOR (2017); SANDE \& SANDE (2018); CANI et } \\
\text { al. (2017); PAGANINI \& BOLZAN (2016); LOPES } \\
\text { \& OLIVEIRA (2013); MARQUES, SILVA \& } \\
\text { MARQUES (2011) }\end{array}$ \\
\hline $\begin{array}{l}\text { Grande parcela dos estudantes já teve ou tem } \\
\text { contato com games }\end{array}$ & $54 \%$ & $\begin{array}{l}\text { ORLANDI et al. (2018); MATTAR \& NESTERIUK } \\
\text { (2016); FRAGELLI (2017); MARTINS et al. (2016); } \\
\text { CHRISTIANINI, DE GRANDE \& AMÉRICO } \\
\text { (2016); MASSI (2017); SANDE \& SANDE (2018); } \\
\text { ALTHAUS, DULLIUS \& AMADO (2016); SILVA } \\
\text { (2015); CANI et al. (2017); LOPES \& OLIVEIRA } \\
\text { (2013); MARQUES, SILVA \& MARQUES (2011) }\end{array}$ \\
\hline Sensação de intervalo da vida cotidiana & $14 \%$ & $\begin{array}{l}\text { BUENO \& BIZELLI (2014); CRUZ JUNIOR (2014); } \\
\text { CRUZ JUNIOR (2017) }\end{array}$ \\
\hline $\begin{array}{l}\text { Promove espaços de convivência híbridos, } \\
\text { multimodais e penetrantes }\end{array}$ & $9 \%$ & SCHLEMMER (2016); MARTINS et al. (2016) \\
\hline Pode minimizar a grande evasão escolar & $4 \%$ & RIBEIRO \& MONTANARO (2017) \\
\hline É uma metodologia ativa & $14 \%$ & $\begin{array}{l}\text { MATTAR \& NESTERIUK (2016); FRAGELLI } \\
\text { (2017); MARQUES, SILVA \& MARQUES (2011) }\end{array}$ \\
\hline
\end{tabular}

Fonte: Elaborado pelos autores.

Dentre os estudos revisados, $54 \%$ alegam que a importância de pesquisar a gamificação no contexto educacional se sustenta no fato de ser uma realidade do mundo atual e porque grande parcela dos estudantes já teve ou tem contato com games. 
No que tange à terceira indagação (o artigo possui algum trecho que trata sobre: "Por que a gamificação deve ser utilizada como recurso didático na educação?"), são inframencionadas as respostas encontradas na Tabela 3.

Tabela 3 - Respostas da terceira indagação

\begin{tabular}{|c|c|c|}
\hline Paráfrase das principais respostas & $\%$ & Autores/Ano \\
\hline Aspecto lúdico integrado na gamificação & $59 \%$ & $\begin{array}{l}\text { COELHO (2011); BUENO \& BIZELLI (2014); } \\
\text { CRUZ JUNIOR (2014); RIBEIRO \& MONTANARO } \\
\text { (2017); MATTAR \& NESTERIUK (2016); } \\
\text { AMÉRICO, NAVARI \& BELDA (2014); MARTINS } \\
\text { et al. (2016); CRUZ JUNIOR (2017); MASSI (2017); } \\
\text { SANDE \& SANDE (2018); ALTHAUS, DULLIUS \& } \\
\text { AMADO (2016); CANI et al. (2017); MARQUES, } \\
\text { SILVA \& MARQUES (2011). }\end{array}$ \\
\hline Divertimento & $23 \%$ & $\begin{array}{l}\text { ORLANDI et al. (2018); BUENO \& BIZELLI (2014); } \\
\text { CHRISTIANINI, DE GRANDE \& AMÉRICO } \\
\text { (2016); CANI et al. (2017); CANI et al. (2017) }\end{array}$ \\
\hline Gera prazer & $36 \%$ & $\begin{array}{l}\text { ORLANDI et al. (2018); BUENO \& BIZELLI (2014); } \\
\text { RIBEIRO \& MONTANARO (2017); FRAGELLI } \\
\text { (2017); MARTINS et al. (2016); MONTE, } \\
\text { BARRETO \& ROCHA (2017); MASSI (2017); CANI } \\
\text { et al. (2017) }\end{array}$ \\
\hline Auxilia na solução de problemas & $36 \%$ & $\begin{array}{l}\text { ORLANDI et al. (2018); BUENO \& BIZELLI (2014); } \\
\text { SCHLEMMER (2016); AMÉRICO, NAVARI \& } \\
\text { BELDA (2014); CHRISTIANINI, DE GRANDE \& } \\
\text { AMÉRICO (2016); MASSI (2017); ALTHAUS, } \\
\text { DULLIUS \& AMADO (2016); LOPES \& OLIVEIRA } \\
\text { (2013) }\end{array}$ \\
\hline Auxilia no processo de ensino-aprendizagem & $82 \%$ & $\begin{array}{l}\text { ORLANDI et al. (2018); BUENO \& BIZELLI (2014); } \\
\text { SCHLEMMER (2016); MATTAR \& NESTERIUK } \\
\text { (2016); SAMPAIO \& BERNARDINO (2017); } \\
\text { FRAGELLI (2017); AMÉRICO, NAVARI \& BELDA } \\
\text { (2014); MARTINS et al. (2016); MONTE, } \\
\text { BARRETO \& ROCHA (2017); CRUZ JUNIOR } \\
\text { (2017); CHRISTIANINI, DE GRANDE \& } \\
\text { AMÉRICO (2016); MASSI (2017); SANDE \& } \\
\text { SANDE (2018); ALTHAUS, DULLIUS \& AMADO } \\
\text { (2016); SILVA (2015); CANI et al. (2017); LOPES \& } \\
\text { OLIVEIRA (2013); MARQUES, SILVA \& } \\
\text { MARQUES (2011). }\end{array}$ \\
\hline Gera motivação & $64 \%$ & $\begin{array}{l}\text { ORLANDI et al. (2018); SCHLEMMER (2016); } \\
\text { MATTAR \& NESTERIUK (2016); SAMPAIO \& } \\
\text { BERNARDINO (2017); FRAGELLI (2017); } \\
\text { AMÉRICO, NAVARI \& BELDA (2014); MARTINS } \\
\text { et al. (2016); CHRISTIANINI, DE GRANDE \& } \\
\text { AMÉRICO (2016); MASSI (2017); SILVA (2015); } \\
\text { CANI et al. (2017); PAGANINI \& BOLZAN (2016); } \\
\text { LOPES \& OLIVEIRA (2013); MARQUES, SILVA \& } \\
\text { MARQUES (2011) }\end{array}$ \\
\hline Propulsiona desafios & & $\begin{array}{l}\text { ORLANDI et al. (2018); BUENO \& BIZELLI (2014); } \\
\text { CRUZ JUNIOR (2014); RIBEIRO \& MONTANARO } \\
\text { (2017); MATTAR \& NESTERIUK (2016); } \\
\text { SAMPAIO \& BERNARDINO (2017); AMÉRICO, } \\
\text { NAVARI \& BELDA (2014); MONTE, BARRETO \& } \\
\text { ROCHA (2017); MASSI (2017); LOPES \& }\end{array}$ \\
\hline
\end{tabular}




\begin{tabular}{|c|c|c|}
\hline & $50 \%$ & $\begin{array}{l}\text { OLIVEIRA (2013); MARQUES, SILVA \& } \\
\text { MARQUES (2011) }\end{array}$ \\
\hline Desenvolve competência e habilidades & $32 \%$ & $\begin{array}{l}\text { SCHLEMMER (2016); MATTAR \& NESTERIUK } \\
\text { (2016); CRUZ JUNIOR (2017); MASSI (2017); } \\
\text { CANI et al. (2017); LOPES \& OLIVEIRA (2013); } \\
\text { MARQUES, SILVA \& MARQUES (2011). }\end{array}$ \\
\hline $\begin{array}{l}\text { Os alunos preferem as aulas gamificadas às } \\
\text { tradicionais }\end{array}$ & $4 \%$ & SAMPAIO \& BERNARDINO (2017) \\
\hline Eleva a produtividade da aula & $4 \%$ & MONTE, BARRETO \& ROCHA (2017) \\
\hline
\end{tabular}

Fonte: Elaborado pelos autores.

Dentre as principais respostas encontradas, os autores corroboram com o uso da gamificação como recurso didático na educação porque: auxilia no processo de ensinoaprendizagem (82\%), motivação (62\%), aspecto lúdico integrado na gamificação (59\%) e por propulsionar desafios $(50 \%)$.

\section{Conclusão e proposta de agenda de pesquisa}

Neste trabalho, procuramos promover reflexões sobre a origem e a conceituação da gamificação, a importância de pesquisar a gamificação no contexto educacional e revelar por que a gamificação é utilizada como recurso didático na educação, com base em periódicos indexados na plataforma de diretórios de periódicos CAPES/MEC, tentando demonstrar que ainda há muito para se pesquisar nessa temática e muito a se fomentar no que tange ao uso da gamificação em todos os níveis e modalidades de ensino.

A gamificação enquanto função educativa é um excelente método alternativo para propiciar o ensino-aprendizagem do educando, por ser uma metodologia ativa, com resultados positivos em todos os artigos revisados que aplicaram metodologias com gamificação no ambiente escolar. Além disso, a gamificação é uma importante ferramenta permeada por momentos de divertimento, motivação e prazer, impactando em um aumento de produtividade em sala de aula. No entanto, a efetiva utilização de metodologias de ensino-aprendizagem com as atividades gamificadas, na prática docente, é um desafio no processo educacional, pois a falta de professores capacitados e atualizados para usar a gamificação no cotidiano escolar cria uma forte resistência em sua aplicabilidade. Sabendo-se que inúmeras pesquisas comprovam que o uso da gamificação tornam as aulas mais produtivas e motivadoras, deveria, por esse motivo, ser mais um método alternativo adotado por todos os docentes que buscam excelência na educação.

Como recomendação geral, pesquisas futuras poderiam realizar maiores estudos para delimitar claramente as categorizações ou espécies de gamificação, considerando uma confusão generalizada entre diversos autores quando utilizavam termos similares a gamificação, como: jogos, jogos digitais, games, videogames, gameful design, edutretenimento, advergames, serious games, simuladores virtuais, laboratórios virtuais e realidade virtual. Pode-se inferir da leitura dos artigos revisados que diversos autores utilizaram nomenclaturas incorretas, justamente pela ausência de uma conceituação clara de cada termo acima citado.

Dentre as questões mais relevantes da pesquisa que ficaram em aberto, vale citar pontos da metodologia: a) não foram utilizados livros para as três indagações elaboradas, o que pode ter deixado a desejar sobre o ponto de vista da opinião dos autores clássicos da área; b) não foram utilizadas dissertações e teses no presente trabalho, o que poderia contribuir com o trabalho. 
No que tange às perspectivas futuras sobre a gamificação, espera-se que seja a cada dia mais utilizada pelos docentes em suas aulas como um método alternativo de ensino aprendizagem, resultando em uma metodologia ativa, motivada e interessante na visão dos discentes.

Espera-se ainda, para o futuro, que a gamificação seja tratada como uma "ciência" na Plataforma Sucupira (Capes), perdendo sua característica de uma subárea dos periódicos das áreas de educação, ensino, comunicação e informação, ciência da computação, interdisciplinar, linguística e literatura e medicina. Afinal, umas das consequências disso, para a área, é a perda de espaço por omissão, ou seja, um tema que deveria ser desenvolvido essencialmente no âmbito da "ciência da gamificação" acaba se desenvolvendo em outras áreas.

Os objetivos específicos do presente trabalho diziam respeito a: a) efetuar revisão bibliográfica sobre a conceituação de gamificação; b) verificar a importância de pesquisar a gamificação no contexto educacional; c) identificar o por que a gamificação deve ser utilizada como recurso didático na educação.

A metodologia adotada na presente pesquisa conseguiu atender a todos os objetivos. Todavia, conforme supracitado, pesquisas futuras poderiam realizar maiores estudos para delimitar claramente a conceituação de termos similares a gamificação, como: jogos, jogos digitais, games, videogames, gameful design, edutretenimento, advergames, serious games, simuladores virtuais, laboratórios virtuais e realidade virtual.

\section{Referências}

ALMEIDA, C.M.M.; PROCHNOW, T.R.; LOPES, P.T.C. O uso do lúdico no ensino de ciências: jogo didático sobre a química atmosférica. Góndola, Enseñ Aprend Cienc, v. 11, n. 2, pp. 228-239, 2016.

ALVES, L.G.; SILVA, J.B.L. Jogos e brincadeiras como estratégia de Ensino-aprendizagem. Revista Eventos Pedagógicos, v. 5, n.4 (13. ed.), número regular, pp. 56-64, nov./dez. 2014.

ANDRADE, N.; QUESADA, R.; MARTINS, V. Jogos digitais no ensino de língua portuguesa e literatura: uma nova possibilidade. Revista Sistemas, Cibernética e Informática, v. 13, n. 3, 2016.

ALTHAUS, N.; DULLIUS, M.D.; AMADO, N.M.P. Jogo computacional e resolução de problemas: três estudos de casos. Revista Educação Matemática Pesquisa, v. 18, n.1, pp. 17-42, 2016.

AMÉRICO, M.; NAVARI, S.C.; BELDA, F.R. Gamificação: modelagem de conteúdos para programas e aplicativos educativos no ambiente da TV digital interativa. Revista IberoAmericana de Estudos em Educação, v. 9, n.4, pp. 804-821, 2014.

BUENO, C.J.S; BIZELLI, J.L. A gamificação do processo educativo. Revista Geminis, v. 5, n.2, pp. 160-176, 2014.

BERTON, M.; USTÁRROZ, C.P.; COSTA, B.F. Ludicidade e turismo: um jogo para apresentar roteiros de turismo gastronômico na cidade de pelotas. Revista Applied Tourism, v. 1, n.3, pp. 1-10, 2016. 
CANI, J.B.; PINHEIRO, I.Q.; SANTIAGO, M.E.V.; SOARES, G.M. Análise de jogos digitais em dispositivos móveis para aprendizagem de línguas estrangeiras. Revista Brasileira de Linguística Aplicada, v. 17, n. 3, pp. 455-481, 2017.

CARVALHO, L.A; WAGNER, L.A.N; QUITETE, T.M.C. O corpo e o universo lúdico no desenvolvimento de habilidades essenciais no processo de letramento e alfabetização. Revista Perspectivas online: hum. \& sociais aplicadas, v. 7, n. 3, pp. 69-78, 2013.

CIESLAK, I. A. Discover Everything you Need to Know about Games and Non-games. North Charleston: Createspace Independent Publishing, 2018.

COELHO, P.M.F. Um mapeamento do conceito de jogo. Revista Geminis, v. 2, n.1, pp. 293$311,2011$.

COMITÊ GESTOR DA INTERNET NO BRASIL. Pesquisa sobre o uso da Internet por crianças e adolescentes. São Paulo: ICT Kids Online Brasil, 2013.

CHRISTIANINI, S.N.; DE GRANDE, F.C.; AMÉRICO, M. Desenvolvimento de sistemas gamificados com foco no edutretenimento e no jogador: uma análise dos arquétipos de Bartle e Marczewski. Revista Ibero-Americana de Estudos em Educação, v. 11, n. esp. 1, pp. 363-373, 2016.

CRUZ JUNIOR, G. Burlando o círculo mágico: o esporte no bojo da gamificação. Revista Movimento, v. 20, n. 3, pp. 941-963, jul./set. 2014.

CRUZ JUNIOR, G. Vivendo o jogo ou jogando a vida? Notas sobre jogos (digitais) e educação em meio à cultura ludificada. Revista Brasileira de Ciências do Esporte, v. 39, n. 3, pp. 226-232, 2017.

DETERDING, S.; DIXON, D. KHALED, R.; NACKE, L. From Game Design Elements to Gamefulness: Defining Gamification. Proceedings of the 15th International Academic MindTrek Conference: Envisioning Future Media Environments, pp. 9-15, 2011.

DOMINGUEZ, A., SAENZ-DE-NAVARRETE, J., DE-MARCOS, L., FERNINDEZ-SANZ, L., PAGES, C., MARTINEZ-HERRIZ, J. J. Gamifying Learning Experiences: Practical Implications and Outcomes. Computers \& Education, v. 63, pp. 380-392, 2013.

FRAGELLI, T.B.O. Gamificação como um processo de mudança no estilo de ensino aprendizagem no ensino superior: um relato de experiência. Revista Internacional de Educação Superior, v.4, n.1, pp. 221-233, jan./abr. 2017.

FURLAN, S.A.; LIMA, J.M.; LIMA, M.R.C.; GUEDES, C.R. Ludicidade e mediações televisivas no contexto escolar. Revista Colloquium Humanarum, v. 12, n.4, pp. 55-68, out./dez. 2015.

GOMES, M. V. M.; PEREIRA-GUIZZO, C. S.; SAMPAIO, R. R.; NASCIMENTO, J. O. Jogos digitais para o desenvolvimento da habilidade espacial: uma revisão da literatura internacional. Revista Educação: Teoria e Prática, v. 28, n. 58, pp. 357-373, mai./ago. 2018. 
GROSSI, M.G.R. O lúdico na aprendizagem de alunos de cursos técnico, graduação e mestrado: relatos de experiências. Revista Ibero-Americana de Estudos em Educação, v. 12, n. 3, pp. 1689-1709, jul./set. 2017.

HANSTED, T.C.; GOHN, M.G. Teatro e educação: uma relação historicamente construída. Eccos Revista Científica, n. 30, pp. 199-220, jan./abr. 2013.

LANDERS, R. N. Developing a Theory of Gamified Learning: Linking Serious Games and Gamification of Learning. Simulation \& Gaming, v. 45, n. 6, pp. 752-768, 2014.

LOPES, N.; OLIVEIRA, I. Videojogos, serious games e simuladores na educação; usar, criar e modificar. Revista Educação, Formação \& Tecnologias, v. 6, n.1, pp. 4-20, jul. 2013.

MARQUES, A.; SILVA, B.D.; MARQUES, N. A influência dos videojogos no rendimento escolar dos alunos: uma experiência no $2^{\circ}$ e $3^{\circ}$ Ciclo do ensino básico. Revista Educação, Formação \& Tecnologias, v. 4, n. 1, pp. 17-27, mai. 2011.

MARTINS, C.; GIRAFFA, L. M. M. Design de práticas pedagógicas incluindo elementos de jogos digitais em atividades gamificadas. Revista Obra Digital, n. 10, pp. 52-67, fev. 2016.

MARTINS, D.M.; BOTTENTUIT JUNIOR, J.B; MARQUES, A.A; SILVA, N.M. A gamificação no ensino de história: o jogo "Legend of zelda" na abordagem sobre medievalismo. Revista Holos, v. 32, n. 7, pp. 299-321, 2016.

MASSI, M.L.G. Criação de objetos de aprendizagem gamificados para uso em sala de treinamento. Revista Científica Hermes, n. 17, pp. 18-35, jan./abr. 2017.

MATTAR, J.; NESTERIUK, S. Estratégias do design de games que podem ser incorporadas à educação a distância. Revista Iberoamericana de Educación a Distancia, v. 19, n. 2, pp. 91-106, 2016.

MEDEIROS, R. A.; LIMA, R.; SILVA, D.; MERCADO, L. P. Jogos digitais como estratégia de ensino-aprendizagem no ensino superior: a construção e aplicação do jogo "Renascença" na disciplina de literatura. Revista Obra Digital, n. 10, pp. 69-83, fev. 2016.

MENDONÇA, S.R.P. A matemática nas turmas de proeja: o lúdico como facilitador da aprendizagem. Revista Holos, Ano 26, Vol. 3, pp. 136-149, 2010.

MONTE, W.S.; BARRETO, M.M.; ROCHA, A.B. Gamification e a web 2.0: planejando processo ensino-aprendizagem. Revista Holos, v. 32, n. 3, pp. 90-97, 2017.

ORLANDI, T.R.C.; DUQUE, C.G.; MORI, A.M. Gamificação: uma nova abordagem multimodal para a educação. Revista Biblios, v. 70, pp. 17-30, 2018.

PAULA, B. H.; VALENTE, J. A. Jogos digitais e educação: uma possibilidade de mudança de abordagem pedagógica no ensino formal. Revista Ibero-americana de Educação, v. 70, n. 1, pp. 9-28, jan. 2016. 
PAGANINI, Érico Rodrigues; BOLZAN, Márcio de Souza. Ensinando física através da gamificação. Blucher Physics Proceedings, v. 3, n.1, pp. 16-20, 2016.

RIBEIRO, L.R; MONTANARO, P.R. A gamificação gamificada: desenvolvimento de um curso para capacitação de docentes. Revista on line de Política e Gestão Educacional, v. 21, n. esp.3, pp. 1625-1637, dez. 2017.

RICHTER, G.; RABAN, D. R.; RAFAELI S. Studying Gamification: the Effect of Rewards and Incentives on Motivation. Gamification in Education and Business (pp. 21-46). Cham: Springer, 2015.

SAMPAIO, D.B.; BERNARDINO, M.C.R. O uso de metodologias alternativas no ensino de biblioteconomia: gamificação como estratégia pedagógica. Revista Brasileira de Educação em Ciência da Informação, v. 4, n. esp., pp. 100-117, 2. sem. 2017.

SANDE, D.; SANDE, D. Uso do Kahoot como ferramenta de avaliação e ensinoaprendizagem no ensino de microbiologia industrial. Revista Holos, v. 34, n. 1, pp. 170-179, 2018.

SCHLEMMER, E. Games e gamificação: uma alternativa aos modelos de EaD. Revista Iberoamericana de Educación a Distancia, v. 19, n. 2, pp. 107-124, 2016.

SILVA, J.C.L. Uso de gamificação como instrumento de avaliação da aprendizagem. Revista FATEC Zona Sul, v. 1, n. 2, pp. 19-31, 2015.

SOARES, E.L.; VIÇOSA, C.S.C.L.; TAHA, M.S.; FOLMER, V. A presença do lúdico no ensino dos modelos atômicos e sua contribuição no processo de ensino aprendizagem.

Revista Góndola, Enseñanza y Aprendizaje de lãs Ciencias. v.12, n. 2 (jul-dez), pp. 69-80, 2017.

VIEIRA, M.S. Dança e a proposta da transdisciplinaridade na Educação. Eccos Revista Científica, n. 27, pp. 55-65, jan./abr. 2012.

Recebido em setembro de 2019.

Aprovado em maio de 2020. 